\section{Effectiveness of International Travel Controls for Delaying Local Outbreaks of COVID-19}

\author{
Bingyi Yang, Sheena G. Sullivan, Zhanwei Du, \\ Tim K. Tsang, Benjamin J. Cowling
}

Author affiliations: The University of Hong Kong, Hong Kong, China (B. Yang, Zhanwei Du, T.K. Tsang, B.J. Cowling); University of Melbourne Peter Doherty Institute for Infection and Immunity, Melbourne, Victoria, Australia (S.G. Sullivan); Laboratory of Data Discovery for Health Limited, Hong Kong (Z. Du, B.J. Cowling)

DOI: https://doi.org/10.3201/eid2801.211944

During the coronavirus disease pandemic, international travel controls have been widely adopted. To determine the effectiveness of these measures, we analyzed data from 165 countries and found that early implementation of international travel controls led to a mean delay of 5 weeks in the first epidemic peak of cases.

Tnternational travel control (e.g., screening of inLbound travelers, requiring quarantines, and even closing borders) has been a key strategy implemented by many countries to limit importations of severe acute respiratory syndrome coronavirus 2 (SARS$\mathrm{CoV}-2)$. However, early in the coronavirus disease (COVID-19) pandemic, the World Health Organization (WHO) did not recommend restricting travel (1), and travel controls have not been widely used in previous pandemics (e.g., the 2009-10 influenza pandemic) $(2,3)$. Limiting international movement has enormous social and economic costs, and the benefits of this strategy (i.e., delaying or averting an epidemic) lack real-world evidence. Previous studies, most of which were simulation studies, suggest that travel restrictions can delay but not prevent local epidemics (2-4).

To examine the association between implementation of international travel controls and local outbreak progress of COVID-19, we used publicly available data (5-7; T. Wu et al., unpub. data, https://www. medrxiv.org/content/10.1101/2020.02.25.2002743 $3 v 1$ ) for January 1-July 31, 2020. Only 14 (8.5\%) of the 165 countries studied enacted international travel controls coincident with the lockdown in Wuhan, China (January 23); all controls involved screening inbound travelers (Figure). Enactment of international travel controls peaked $\approx 3$ weeks after WHO declared the pandemic (March 11, 2020), by which time $112(67.8 \%)$ countries completely closed their borders, 44 (26.6\%)

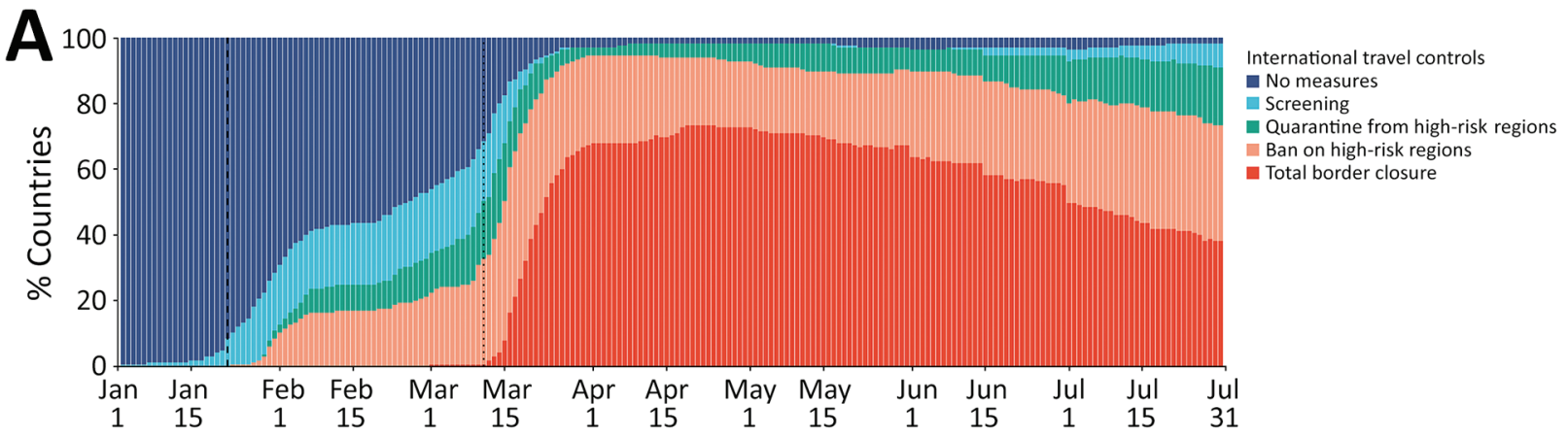

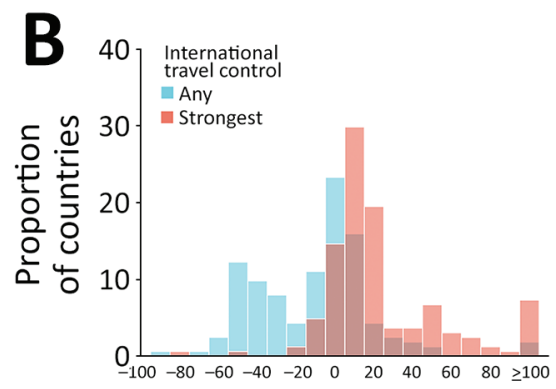

Days in relation to first case
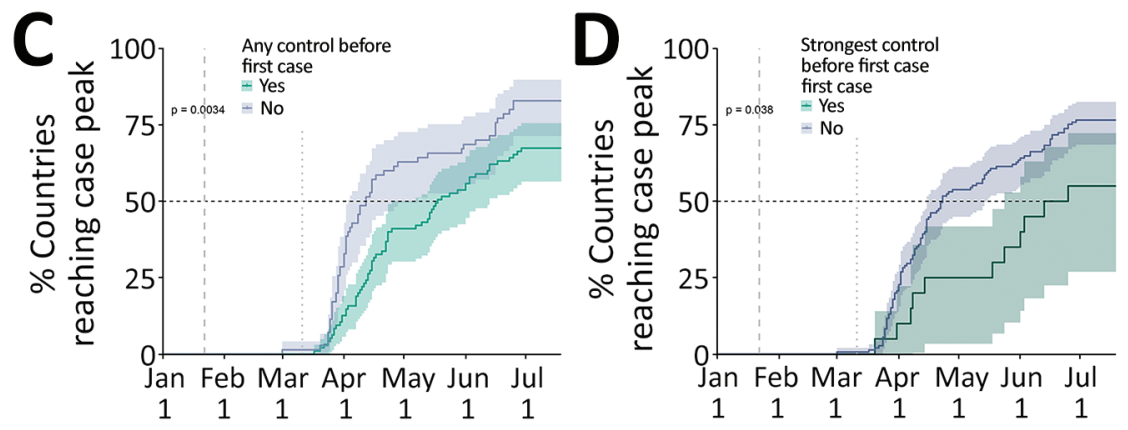

Figure. Association between international travel controls and local coronavirus disease (COVID-19) outbreaks in 165 countries, January 1-July 31, 2020. A) Temporal distribution of the international travel controls enacted by the studied countries. Data from (7). B) Distribution of the time between a country's first COVID-19 case and its enactment of any or of the strongest international travel controls. C, D) Probability of reaching first local peak of COVID-19 cases by the time of implementing any (C) or the strongest (D) international travel controls, estimated by using the Kaplan-Meier survival function. Vertical dashed lines in panels B, C, and D indicate the date that Wuhan, China, underwent lockdown; vertical dotted lines indicate the date that the pandemic was declared. 
Table. Estimated time ratios and hazard ratios for comparing selected outcomes in countries that did and did not implement international controls before identifying their first cases of COVID-19, January-July 2020 *

\begin{tabular}{|c|c|c|c|c|}
\hline \multirow[b]{2}{*}{ Endpoint } & \multicolumn{2}{|c|}{ Adjusted time ratio $(95 \% \mathrm{Cl}) \dagger$} & \multicolumn{2}{|c|}{ Adjusted hazard ratio $(95 \% \mathrm{Cl}) \ddagger$} \\
\hline & Any international controls & $\begin{array}{l}\text { The strongest } \\
\text { international controls }\end{array}$ & Any international controls & $\begin{array}{c}\text { The strongest } \\
\text { international controls }\end{array}$ \\
\hline Case peak & $1.22(1.06-1.41)$ & $1.31(1.02-1.68)$ & $0.66(0.46-0.93)$ & $0.65(0.39-1.08)$ \\
\hline Death peak & $1.23(1.01-1.51)$ & $0.98(0.71-1.37)$ & $0.74(0.53-1.04)$ & $0.90(0.53-1.55)$ \\
\hline \multicolumn{5}{|c|}{ Cumulative incidence, no. cases $/ 10,000$ population } \\
\hline 0.2 & $1.20(1.10-1.31)$ & $1.23(1.05-1.44)$ & $0.55(0.38-0.78)$ & $0.61(0.35-1.04)$ \\
\hline 1.0 & $1.26(1.13-1.42)$ & $1.27(1.04-1.55)$ & $0.49(0.35-0.71)$ & $0.90(0.53-1.51)$ \\
\hline 5.0 & $1.25(1.05-1.49)$ & $1.34(0.99-1.82)$ & $0.59(0.41-0.85)$ & $0.90(0.54-1.51)$ \\
\hline \multicolumn{5}{|c|}{$\begin{array}{l}\text { *AFT, accelerated failure time; COVID-19, coronavirus disease. } \\
\text { †Estimates were obtained from accelerated failure time models with log-logistic distribution, adjusted for population density and the strictest level of each } \\
\text { nonpharmaceutical intervention used during the study period for each country. The } 2 \text { columns show time ratio of implementing international controls } \\
\text { before the country's first COVID-19 case to that after the country's first case. } \\
\text { fEstimates were obtained from Cox proportional hazard models, which adjusted for population density and time-varying nonpharmaceutical interventions } \\
\text { during the study period for each country. The } 2 \text { columns show hazard ratio of implementing international controls before the country's first COVID-19 case } \\
\text { to that after the country's first case. }\end{array}$} \\
\hline
\end{tabular}

banned travelers from high-risk regions, and 4 (2.4\%) required quarantine for travelers from high-risk regions (Figure; Appendix Figure 1, https://wwwnc. cdc.gov/EID/article/28/1/21-1944-App1.pdf). Of the 165 countries, $90(54.5 \%)$ had imposed at least some restriction before reporting their first COVID-19 case, and $20(12 \%)$ had imposed their strictest restrictions before reporting their first case (Figure; Appendix Figures 1-3).

We determined the progress of outbreaks in each country to be the time from January 1,2020, to the first epidemic peak, which was identified from the modal daily case counts within any 53-day sliding window (i.e., a quarter of the length of the study period) and needed to comprise $\geq 10 \%$ of the cumulative incidence during the study period (Appendix Figure 2). By July 31,2020 , the first epidemic peak had been reached in $122(74 \%)$ of the studied countries (Appendix Figure 4). In countries that had enacted any international travel controls before their first COVID-19 case, the first peak was reached an average of 36 days (95\% CI 10-61 days) later than it was in countries that did not enact controls until after their first case was reported $(p<0.01$ by log-rank test; Figure). Countries that implemented their strictest international travel controls before detecting any COVID-19 cases reported their first case a median of 57 days (95\% CI 14-70 days) later than countries that imposed their strongest controls after the first case was reported ( $p=0.04$ by logrank test; Figure).

After adjusting for population density and implementing nonpharmaceutical interventions by using the accelerated failure time model (Appendix), we estimated that the average time to detection of the first case occurred 1.22 (95\% CI 1.06-1.41) times later in countries that implemented any restrictions than in countries that implemented no travel restrictions. This time ratio was extended to 1.31 (95\% CI 1.02-
1.68) if countries implemented their strongest travel restrictions (Table). Such associations still held when adjusting for time-varying nonpharmaceutical interventions by using the Cox model.

To confirm that these observations were maintained according to alternative measures of epidemic activity, we used the following as outcomes in the models: the time by which COVID-19 deaths first peaked, and attainment of a cumulative incidence of 0.2 , 1.0, or 5.0 cases $/ 10,000$ persons (by which time peaks had been reached in $\approx 10 \%, 30 \%$, and $60 \%$ of the countries; Appendix Figure 5). These outcomes may better indicate community spread in countries in which most cases were imported and identified during quarantine (e.g., Fiji), information that was not available from public data. Moreover, outcomes may be better when the epidemic was multimodal (e.g., Guyana) or the country did not experience its main epidemic until later in the study period (e.g., Argentina) (Appendix Figure 2). Both accelerated failure time and Cox models supported earlier observations that enactment of any international travel controls delayed the time in which cumulative incidence rates or deaths peaked. However, enactment of the strongest control was not associated with a reduced time to peak death or cumulative incidence of 5 cases $/ 100,000$ persons (Table).

Our work may be influenced by other unmeasured confounders, such as the stringency of international travel controls. We repeated our analyses by removing countries in Asia, in which implementation tended to be more strict, and found that our earlier observations largely held (Appendix Table). In addition, we examined the broader association between international travel controls and local epidemic progression, but we did not examine the roles of specific measures (e.g., quarantine and risk-dependent triage management). 
Our findings suggest that implementing international travel controls earlier delayed the initial epidemic peak by $\approx 5$ weeks. Although travel restrictions did not prevent the virus from entering most countries, delaying its introduction bought valuable time for local health systems and governments to prepare to respond to local transmission.

\section{Acknowledgments}

We thank the Department of Health of the Food and Health Bureau of the Government of Hong Kong for conducting the outbreak investigation and providing data for analysis.

This project was supported by the Health and Medical Research Fund, Food and Health Bureau and Government of the Hong Kong Special Administrative Region (grant no. COVID190118). The WHO Collaborating Centre for Reference and Research on Influenza is supported by the Australian Government Department of Health.

B.J.C. consults for Roche, GSK, Moderna, AstraZeneca, and Sanofi Pasteur and is supported by the AIR@innoHK program of the Innovation and Technology Commission of the Hong Kong Special Administrative Region Government. S.G.S. reports performing unpaid consulting for Sanofi Pasteur and Sequiris. The authors report no other potential conflicts of interest.

All authors are affiliated with WHO collaborating centers. The objective technical analysis and results reported here were not part of official WHO work, and opinions contained herein do not necessarily represent the views of WHO.

\section{About the Author}

Dr. Yang is a postdoctoral fellow at the School of Public Health, University of Hong Kong. Her research interests are quantifying transmission dynamics and control of infectious diseases.

\section{References}

1. World Health Organization. Statement on the second meeting of the International Health Regulations (2005) Emergency Committee regarding the outbreak of novel coronavirus (2019-nCoV) [cited 2021 Aug 2]. https:/ / www.who.int/news/ item/30-01-2020-statement-on-the-second-meeting-of-theinternational-health-regulations-(2005)-emergency-committeeregarding-the-outbreak-of-novel-coronavirus-(2019-ncov)

2. Cowling BJ, Lau LLH, Wu P, Wong HWC, Fang VJ, Riley S, et al. Entry screening to delay local transmission of 2009 pandemic influenza A (H1N1). BMC Infect Dis. 2010;10:82. https://doi.org/10.1186/1471-2334-10-82

3. Ryu S, Gao H, Wong JY, Shiu EYC, Xiao J, Fong MW, et al. Nonpharmaceutical measures for pandemic influenza in nonhealthcare settings-international travel-related measures. Emerg Infect Dis. 2020;26:961-6. https:/ / doi.org/10.3201/ eid2605.190993
4. Burns J, Movsisyan A, Stratil JM, Coenen M, Emmert-Fees KM, Geffert K, et al. Travel-related control measures to contain the COVID-19 pandemic: a rapid review. Cochrane Database Syst Rev. 2020;10:CD013717

5. Dong E, Du H, Gardner L. An interactive web-based dashboard to track COVID-19 in real time. Lancet Infect Dis. 2020;20:533-4. https:// doi.org/10.1016/S1473-3099 (20)30120-1

6. Hale T, Angrist N, Goldszmidt R, Kira B, Petherick A, Phillips T, et al. A global panel database of pandemic policies (Oxford COVID-19 Government Response Tracker) [cited 2021 Aug 2]. https:/ / www.nature.com/articles/ s41562-021-01079-8

7. Our World in Data. Policy responses to the coronavirus pandemic [cited 2021 Aug 2]. https:/ / ourworldindata.org/ policy-responses-covid

Address for correspondence: Benjamin J. Cowling, School of Public Health, Li Ka Shing Faculty of Medicine, The University of Hong Kong, 7 Sassoon Rd, Pokfulam, Hong Kong, China; email: bcowling@hku.hk

\section{Atezolizumab Treatment for Progressive Multifocal Leukoencephalopathy}

\author{
Nicolas Lambert, Solène Dauby, Dominique Dive, \\ Bernard Sadzot, Pierre Maquet
}

Author affiliation: University Hospital of Liège, Liège, Belgium

DOI: https://doi.org/10.3201/eid2801.204809
Atezolizumab successfully reinvigorated JC virus immunity in a patient in Belgium with progressive multifocal leukoencephalopathy, as demonstrated by clinical, virologic, and radiologic response to treatment. However, the treatment also resulted in immune reconstitution inflammatory syndrome and life-threatening immune-related adverse events. These conditions were treated with corticosteroids, leading to treatment resistance.

P rogressive multifocal leukoencephalopathy (PML) is a devastating infectious disease of the brain that is caused by JC virus (JCV) in the context of cellular immunodeficiency. To date, no effective antiviral treatment for PML exists, and survival depends on the person's ability to achieve timely immune 